\title{
Diffusions with Bessel-like drifts
}

\author{
Yuji Kasahara and Shin'ichi Kotani
}

\begin{abstract}
Linear diffusions which are not far from Bessel diffusions are considered. Specifically, the regular variation of Feller's canonical measure is interpreted in terms of the drift coefficient. As an application, the asymptotic behavior of the transition probability at large times is discussed.
\end{abstract}

\section{Introduction}

Let $X=\left(X_{t}\right)_{t \geq 0}$ be a regular conservative diffusion on $I=[0, \infty)$ with local generator of the form

$$
\mathcal{L}=\frac{1}{2}\left(\frac{d^{2}}{d x^{2}}+b(x) \frac{d}{d x}\right), \quad x>0,
$$

with $b(x)$ being assumed to be an element of $L_{\text {loc }}^{1}((0, \infty), d x)$. We put a reflecting boundary condition at 0 when it is regular. As is well known, $\mathcal{L}$ in (1.1) can be written in Feller's canonical form as

$$
\mathcal{L}=\frac{d}{d m(x)} \frac{d}{d s(x)} .
$$

The increasing continuous function $s(x)$ is called the scale function and the Lebesgue-Stieltjes measure $d m(x)$ is called the speed measure or the canonical measure. For their probabilistic meaning we refer to K. Itô [4] or Itô-McKean [5].

Since many limit theorems for diffusions are given in terms of $\widetilde{m}(x):=$ $m\left(s^{-1}(x)\right)$ (e.g., [7], [9], [13], [14], [12]; see also Theorems A and B in Section 5), it will be of interest to interpret them in terms of $b(x)$. Therefore, the aim of the present article is to discuss the mutual dependency of $\widetilde{m}(x)$ and $b(x)$.

As a typical application of this problem we shall also study the asymptotic behavior of the transition density in the long term. A diffusion process on $[0, \infty)$ associated with

$$
\mathcal{L}_{\rho}=\frac{1}{2}\left(\frac{d^{2}}{d x^{2}}+\frac{\rho-1}{x} \frac{d}{d x}\right), \quad x>0, \rho>0
$$

Kyoto Journal of Mathematics, Vol. 55, No. 4 (2015), 773-797 DOI 10.1215/21562261-3157739, (C) 2015 by Kyoto University

Received November 21, 2013. Revised September 4, 2014. Accepted September 16, 2014.

2010 Mathematics Subject Classification: Primary 60J60; Secondary 34B24.

Kasahara's work was supported by Japan Society for the Promotion of Science Grant-in-Aid for Scientific Research number 24540110. 
is called a $\rho$-dimensional Bessel process or a Bessel process of order $\nu:=(\rho / 2)-1$. We put the reflecting boundary condition at 0 when it is regular (i.e., $0<\rho<2$ ). The transition density $p_{\rho}(t, x, y)$ with respect to the speed measure $m_{\rho}(d x):=$ $2 x^{\rho} d x$ is

$$
p_{\rho}(t, x, y)=\frac{1}{2 t}(x y)^{-\nu} \exp \left(-\frac{x^{2}+y^{2}}{2 t}\right) I_{\nu}\left(\frac{x y}{t}\right),
$$

where $I_{\nu}(x)$ is the modified Bessel function (see, e.g., Borodin-Salminen [2, pp. 133-134]). Since

$$
I_{\nu}(x) \sim \frac{1}{\Gamma(\nu+1)}\left(\frac{x}{2}\right)^{\nu} \quad(x \rightarrow 0)
$$

it holds that

$$
p_{\rho}(t, x, y) \sim \frac{1}{2^{\rho / 2} \Gamma(\rho / 2)} \frac{1}{\sqrt{t}^{\rho}} \quad(t \rightarrow \infty) .
$$

Here, ' $f(x) \sim c g(x)$ ' denotes ' $f(x) / g(x) \rightarrow c$ ' for $c \in \mathbb{R}$.

Now we return to (1.1) and let $p(t, x, y)$ be its transition density with respect to the speed measure (see Section 4 for details). Let us generalize (1.4) for more general diffusions. Since such a problem has been discussed mainly in terms of $\widetilde{m}(x)$ as we mentioned above, our interest here is to interpret them in terms of $b(x)$.

Our main result is as follows. If

$$
b(x) \sim \frac{\rho-1}{x} \quad(x \rightarrow \infty),
$$

then

$$
p(t, x, y) \sim \frac{1}{2^{\rho / 2} \Gamma(\rho / 2)} \frac{1}{\sqrt{t}} \exp \left\{-\int_{1}^{\sqrt{t}} b(x) d x\right\} \quad(t \rightarrow \infty) .
$$

This can also be written as follows (see Theorem 4.2): if $\mathcal{L}=\mathcal{L}_{\rho}+q(x) \frac{d}{d x}$ with $q(x)=o(1 / x)$, then

$$
p(t, x, y) \sim p_{\rho}(t, x, y) / L(\sqrt{t}) \quad(t \rightarrow \infty),
$$

where

$$
L(x)=\exp \int_{1}^{x} q(t) d t, \quad x>0 .
$$

What is interesting is that the converse holds under a mild condition (see Theorem 4.3). As a typical example of these results we shall give a necessary and sufficient condition on $b(x)$ for

$$
p(t, x, y) \sim \operatorname{const} \cdot \frac{1}{\sqrt{t}^{\rho}(\log t)^{\beta}} \quad(t \rightarrow \infty),
$$

under a mild condition (see Example 4.1).

The present article is organized as follows. In Section 2 we give a few basic formulas on the relationship between $b$ and $\widetilde{m}$. In Section 3 we discuss the regular variation of $\widetilde{m}$. Theorems 3.1-3.3 are the main theorems. An application is given 
in Section 4, where we discuss (1.5) (or, equivalently, (1.6)), the proof of which will be given in Section 5 . In Section 6 we treat the case of diffusion on the whole line $(-\infty, \infty)$ and give some applications to skew Bessel processes.

\section{Feller's canonical form}

Let us consider a diffusion process on $I=[0, \infty)$ with local generator (1.1) under the condition that $b \in L_{\mathrm{loc}}^{1}((0, \infty), d x)$ and

$$
-1<\liminf _{x \rightarrow+0} x b(x) \leq \limsup _{x \rightarrow+0} x b(x)<\infty .
$$

We denote by $\mathbb{B}$ the totality of such $b$ 's. For $b \in \mathbb{B}$ define

$$
V(x)=\int_{1}^{x} b(u) d u, \quad W(x)=e^{V(x)}, \quad x>0 .
$$

In other words, $W$ is the solution of

$$
\frac{W^{\prime}(x)}{W(x)}=b(x), \quad W(1)=1 .
$$

The initial condition $W(1)=1$ is not essential. The lower bound of the integral in (2.2) may be replaced by any other number. Nonetheless, throughout we shall adopt $(2.2)$ (i.e., $W(1)=1$ ) to avoid the ambiguity of multiplicative constants. When one needs to change this condition, see Remarks 3.2 and 4.1. On the contrary, the condition (2.1) is essential. To treat the case where (2.1) does not hold we need to discuss the bilateral diffusions and we shall not go into such details in the present article.

Next define

$$
s(x)=s(c ; x)=\int_{c}^{x} \frac{1}{W(u)} d u, \quad m(x)=2 \int_{0}^{x} W(u) d u, \quad x \geq 0 .
$$

Here, note that $m(x)$ is finite under the assumption (2.1). The function $s(x)$ depends on the choice of $c$. A most general choice is $c=1$, but it will sometimes be convenient to choose $c=0$ so that $s(0)=0$ when $\int_{0}^{1} e^{-V(u)} d u<\infty$ and $c=\infty$ so that $s(+\infty)=0$ when $\int_{1}^{\infty} e^{-V(u)} d u<\infty$.

We next note that (2.1) implies that the left endpoint 0 of $I$ is an entrance (in the wide sense); that is,

$$
\int_{0}^{1} m(x) d s(x)<\infty
$$

(see Lemma 2.1 for the proof). In terms of $m(x)$ and $s(x)$ the operator $\mathcal{L}$ in (1.1) is written in Feller's canonical form:

$$
\mathcal{L}=\frac{1}{2}\left(\frac{d^{2}}{d x^{2}}+\frac{W^{\prime}(x)}{W(x)} \frac{d}{d x}\right)=\frac{1}{2 W(x)} \frac{d}{d x}\left(W(x) \frac{d}{d x}\right)=\frac{d}{d m(x)} \frac{d}{d s(x)}, \quad x>0 .
$$

We next define, as we mentioned above,

$$
\widetilde{m}(x)= \begin{cases}m\left(s^{-1}(x)\right) & x<\ell \\ +\infty & x \geq \ell\end{cases}
$$


where $\ell=s(\infty)(\leq+\infty)$. Then $\mathcal{L}$ can be written as

$$
\mathcal{L}=\frac{d}{d \widetilde{m}(s)} \frac{d}{d s}, \quad-\infty<s<\ell .
$$

The following function will play a crucial role in the present article:

$$
\widetilde{M}(x):=\int_{-\infty}^{x} \widetilde{m}(u) d u\left(=\int_{0}^{s^{-1}(x)} m(u) d s(u)\right), \quad-\infty<x<\ell .
$$

For $x \geq \ell$ we conventionally define $\widetilde{M}(x)=\infty$. Notice that $\widetilde{M}(x)<\infty$ for $x<\ell$ under our assumption (2.1). In fact, we can say more.

\section{LEMMA 2.1}

(a) We have that (2.1) implies that

$$
\int_{0}^{x} m(y) d s(y)=O\left(x^{2}\right) \quad(x \rightarrow+0) .
$$

(b) If, in addition, for some $C>1$

$$
\limsup _{x \rightarrow+0} x b(x)<C<\infty,
$$

then

$$
\widetilde{M}(x)=O\left(|x|^{-2 /(C-1)}\right) \quad(x \rightarrow-\infty)
$$

and hence

$$
\int_{-\infty}^{-A} \widetilde{M}^{k}(x) d x<\infty, \quad \exists k \geq 1, \exists A>0 .
$$

Proof

(a) There exist $\varepsilon>0$ and $\delta>0$ such that

$$
b(x) \geq \frac{-1+\varepsilon}{x}, \quad 0<x<\delta .
$$

Then,

$$
V(z)-V(y)=-\int_{z}^{y} b(u) d u \leq(1-\varepsilon) \int_{z}^{y} \frac{d u}{u}=(1-\varepsilon) \log \frac{y}{z}
$$

and so

$$
\begin{aligned}
\int_{0}^{x} m(y) d s(y) & =2 \int_{0}^{x} d y \int_{0}^{y} e^{V(z)-V(y)} d z \\
& \leq 2 \int_{0}^{x} d y \int_{0}^{y}\left(\frac{y}{z}\right)^{1-\varepsilon} d z=\frac{x^{2}}{\varepsilon}, \quad 0<x<\delta .
\end{aligned}
$$

(b) By (a) we have that $\left.\widetilde{M}(x)=O\left(s^{-1}(x)^{2}\right)\right)$. Therefore, it remains to show that $s^{-1}(y)=O\left(|y|^{-1 /(C-1)}\right)$ as $y \rightarrow-\infty$. To this end, let $b(x) \leq C / x$ for all $0<x<\delta$. Then,

$$
s(x)=-\int_{x}^{\delta} e^{-V(u)} d u \geq-\int_{x}^{\delta} e^{-C \log u} d u=\frac{\delta^{-C+1}-x^{-C+1}}{C-1}, \quad 0<x<\delta .
$$


Therefore,

$$
s^{-1}(y) \leq\left(\delta^{-C+1}-(C-1) y\right)^{-1 /(C-1)}=O\left(|y|^{-1 /(C-1)}\right) \quad(y \rightarrow-\infty),
$$

which completes the proof.

The scale function $s(x)$ is determined uniquely (up to an additive constant) by $\widetilde{m}(x)$ via the following formula.

\section{LEMMA 2.2}

We have that

$$
\begin{aligned}
\widetilde{m}^{\prime}(x) & =\frac{2}{\left\{s^{\prime}\left(s^{-1}(x)\right)\right\}^{2}}, \\
\sqrt{\widetilde{m}^{\prime}(s(x))} s^{\prime}(x) & =\sqrt{2}, \quad x \in\left(s^{-1}(-\infty), s^{-1}(\infty)\right), \\
\int_{s\left(x_{0}\right)}^{s(x)} \sqrt{\widetilde{m}^{\prime}(u)} d u & =\sqrt{2}\left(x-x_{0}\right), \quad x, x_{0} \in\left(s^{-1}(-\infty), s^{-1}(\infty)\right) .
\end{aligned}
$$

Proof

Since

$$
\widetilde{m}^{\prime}(x)=\left(m\left(s^{-1}(x)\right)\right)^{\prime}=\frac{m^{\prime}\left(s^{-1}(x)\right)}{s^{\prime}\left(s^{-1}(x)\right)}=\frac{2}{\left\{s^{\prime}\left(s^{-1}(x)\right)\right\}^{2}},
$$

we have (2.7). Changing the variable, we obtain (2.8) from (2.7). Then, (2.9) follows from (2.8).

\section{COROLLARY 2.1}

We have that $\widetilde{m}^{\prime}$ determines $W$ (and hence b) uniquely.

Proof

By (2.9), $\widetilde{m}^{\prime}$ determines $s$ (up to an additive constant). Hence, $W=1 / s^{\prime}$ is specified.

\section{EXAMPLE 2.1}

If $\widetilde{m}^{\prime}(x)=2$, then by (2.9) we see that $s(x)-s\left(x_{0}\right)=x-x_{0}$ and hence $W(x)=$ $1 / s^{\prime}(x)=1$. Thus, we have that $b(x)=(\log W(x))^{\prime}=0$.

\section{REMARK 2.1}

When $f(x) \geq 0$ is given, we cannot necessarily find $b(x)$ such that $\widetilde{m}^{\prime}(x)=f(x)$ in general. For example, if $\widetilde{m}^{\prime}(x)=A$ for some constant $A>0$, then we see that $s(x)-s\left(x_{0}\right)=\sqrt{2 / A}\left(x-x_{0}\right), W(x)=\sqrt{A / 2}$, and $V(x)=(1 / 2) \log (A / 2)$. Thus, we have that $b(x)=(\log W(x))^{\prime}=0$ as in Example 2.1. On the other hand, $b(x)=0$ implies that $\widetilde{m}^{\prime}(x)=2$ as we have computed before, and hence $\widetilde{m}^{\prime}(x)=$ $A$ is possible only when $A=2$. This restriction comes from the normalizing condition that $s^{\prime}(1)=1 / W(1)=1$. 
The correspondence $b \leftrightarrow \widetilde{m}$ is continuous in the following sense.

PROPOSITION 2.1 (CONTINUITY)

Let $b_{n}, b \in \mathbb{B}$, and let $s_{n}, s$ and $\widetilde{m}_{n}, \widetilde{m}$ correspond to them, respectively. We normalize $s_{n}(x)$ so that $s_{n}\left(x_{0}\right)=s\left(x_{0}\right)$ for some $x_{0}$. Then

$$
s_{n}(x) \rightarrow s(x), \quad \forall x>0, n \rightarrow \infty,
$$

if and only if

$$
\int_{x_{0}}^{x} \sqrt{\widetilde{m}_{n}^{\prime}(u)} d u \rightarrow \int_{x_{0}}^{x} \sqrt{\widetilde{m}^{\prime}(u)} d u, \quad \forall x, x_{0} \in(s(+0), s(\infty)) .
$$

Proof

Let

$$
F_{n}(x)= \begin{cases}\frac{1}{\sqrt{2}} \int_{x_{0}}^{x} \sqrt{\widetilde{m}_{n}^{\prime}(u)} d u-1 & x>s_{n}(+0), \\ -\infty & x \leq s_{n}(+0) .\end{cases}
$$

Although $s_{n}(x)$ are defined only for $x>0$, we conventionally define $s_{n}(x)=$ $-\infty$ for $x \leq 0$ so that the $s_{n}$ 's are defined on $\mathbb{R}$. Then, since $s_{n}(x)=F_{n}^{-1}(x)$, $F_{n}(x) \Rightarrow F(x)$ if and only if $s_{n}(x) \Rightarrow s(x)$, where $\Rightarrow$ denotes the convergence at the continuity point.

\section{REMARK 2.2}

By Proposition 2.1 we see that $\widetilde{m}_{n}(x) \rightarrow \widetilde{m}(x)$ does not necessarily imply that $s_{n}(x) \rightarrow s(x)$. However, if the $\left\{\widetilde{m}_{n}^{\prime}\right\}_{n}$ 's are monotone functions or if $\widetilde{m}_{n}^{\prime}(x) \rightarrow$ $\widetilde{m}^{\prime}(x)$ holds uniformly on every compact set in $(s(+0), s(\infty))$, then $s_{n}(x) \rightarrow s(x)$.

\section{Regular variation of $\widetilde{M}$ and $W$}

Throughout the article $V(x), W(x), s(x), m(x), \widetilde{m}(x), \widetilde{M}(x)$ corresponding to the Bessel process (1.3) will be denoted by $V_{\rho}(x), W_{\rho}(x), s_{\rho}(x), m_{\rho}(x), \widetilde{m}_{\rho}(x)$, $\widetilde{M}_{\rho}(x)$, respectively; that is, for $x>0$,

$$
V_{\rho}(x)=(\rho-1) \log x, \quad W_{\rho}(x)=x^{\rho-1},
$$

and therefore,

$$
m_{\rho}(x)=\frac{2}{\rho} x^{\rho}, \quad s_{\rho}(x)= \begin{cases}s_{\rho}(0 ; x)=\frac{1}{2-\rho} x^{2-\rho} & 0<\rho<2, \\ s_{\rho}(1 ; x)=\log x & \rho=2, \\ s_{\rho}(\infty ; x)=-\frac{1}{\rho-2} x^{2-\rho} & \rho>2 .\end{cases}
$$

With the convention that $0^{\gamma}=\infty$ if $\gamma<0$, we have that

$$
\tilde{m}_{\rho}(x)= \begin{cases}\frac{2}{\rho}(2-\rho)^{\rho /(2-\rho)}\left(x_{+}\right)^{\rho /(2-\rho)} & 0<\rho<2, \\ e^{2 x} & \rho=2, \\ \frac{2}{\rho}(\rho-2)^{\rho /(2-\rho)}\left(x_{-}\right)^{\rho /(2-\rho)} & \rho>2\end{cases}
$$


and

$$
\widetilde{M}_{\rho}(x)= \begin{cases}\frac{1}{\rho}(2-\rho)^{2 /(2-\rho)}\left(x_{+}\right)^{2 /(2-\rho)} & 0<\rho<2, \\ \frac{1}{2} e^{2 x} & \rho=2, \\ \frac{1}{\rho}(\rho-2)^{2 /(2-\rho)}\left(x_{-}\right)^{2 /(2-\rho)} & \rho>2,\end{cases}
$$

where $x_{+}=\max (x, 0), x_{-}=\max (-x, 0)$.

In what follows let us treat the case where $W(x)$ is not far from $W_{\rho}(x)$. A function $L(x)>0(x>A)$ is said to be slowly varying if

$$
\lim _{x \rightarrow \infty} \frac{L(\lambda x)}{L(x)}=1, \quad \forall \lambda>0 .
$$

Typical examples are const, $\log x$, and $\log \log x$. It has been shown by Karamata [6] that $L(x)>0$ is slowly varying if and only if $L(x)$ may be written in the form

$$
L(x)=c(x) \exp \int_{1}^{x} \frac{\varepsilon(t)}{t} d t
$$

for some measurable $\varepsilon(x) \rightarrow 0$ and $c(x) \rightarrow c>0(x \rightarrow \infty)$ (see, e.g., [1, p. 12]). A measurable function $f(x)>0$ is said to be regularly varying at $\infty$ (or at +0 ) with index $\gamma \in \mathbb{R}$ if and only if

$$
\lim _{x \rightarrow \infty[+0]} \frac{f(\lambda x)}{f(x)}=\lambda^{\gamma}, \quad \forall \lambda>0 .
$$

Clearly, $f(x)$ is regularly varying at $\infty$ with index $\gamma \in \mathbb{R}$ if and only if $f(x) \sim$ $x^{\gamma} L(x)$ for a slowly varying function $L(x)$. Also $f(x)$ is said to be regularly varying at +0 with index $\gamma \in \mathbb{R}$ if and only if $f(1 / x)$ at $\infty$ is as well with index $-\gamma$; that is, $f(x)=x^{\gamma} L(1 / x)$ with slowly varying $L(x)$.

\section{NOTATION}

We denote by $R_{\gamma}(\infty)$ (or $R_{\gamma}(0)$ ) the totality of functions varying regularly at $\infty$ (or at +0 ) with index $\gamma \in \mathbb{R}$.

For $\varphi \in R_{\gamma}(\infty)(\gamma \neq 0)$ the asymptotic inverse $\varphi^{-1}$ can be defined (cf. [1, p. 28]) and $\varphi^{-1} \in R_{1 / \gamma}(\infty)$ or $\varphi^{-1} \in R_{1 / \gamma}(0)$ according to whether $\gamma>0$ or $\gamma<0$.

One of the well-known facts on regularly varying functions is the following: If $f \in R_{\alpha}(\infty)(\alpha>-1)$, then $\int^{x} f(u) d u \sim x f(x) /(\alpha+1) \in R_{\alpha+1}(\infty)$ (see [1, p. 26]). Conversely, if $F \in R_{\alpha}(\infty)(\alpha>0)$ and if $F^{\prime}$ is eventually (i.e., ultimately) monotone, then $F^{\prime}(x) \sim F(x) /(\alpha x) \in R_{\alpha-1}(\infty)$ (monotone density theorem). See $[1$, p. 39] for details. (A function $f(x)$ is said to be eventually nonnegative (or nonpositive) if it is nonnegative (or nonpositive, resp.) for all sufficiently large $x$.)

To rewrite the condition for the regular variation of $W(x)$ in terms of $b(x)$, let us consider the following condition. 


\section{CONDITION 3.1}

The drift coefficient $b \in \mathbb{B}$ has the following representation:

$$
b(x)=\frac{\rho-1+\varepsilon(x)}{x}+\eta(x) \quad(x>1),
$$

where $\varepsilon(x) \rightarrow 0$ and $\int_{1}^{x} \eta(u) d u \rightarrow \bar{\eta} \in \mathbb{R}(x \rightarrow \infty)$.

Since $W(x)=\exp \int_{1}^{x} b(u) d u,(3.3)$ may be written as

$$
W(x)=x^{\rho-1} c(x) \exp \int_{1}^{x} \frac{\varepsilon(u)}{u} d u \quad(x>1),
$$

where $c(x)=\exp \int_{1}^{x} \eta(t) d t$. If we compare this with (3.2) (the canonical representation of slowly varying functions), then we easily have the following.

\section{PROPOSITION 3.1}

We have that $W \in R_{\rho-1}(\infty)$ if and only if Condition 3.1 is satisfied.

A trivial sufficient condition for Condition 3.1 is $b(x) \sim(\rho-1) / x$, in which case just define $\varepsilon(x)=x b(x)-(\rho-1)$ and $\eta(x)=0$.

\section{THEOREM 3.1}

Let $0<\rho<2$, and let $b \in \mathbb{B}$.

(a) $W \in R_{\rho-1}(\infty)$ if and only if $\widetilde{m}^{\prime} \in R_{-(2-2 \rho) /(2-\rho)}(\infty)$.

(b) $W \in R_{\rho-1}(\infty)$ implies that $\widetilde{m} \in R_{\rho /(2-\rho)}(\infty)$ and $\widetilde{M} \in R_{2 /(2-\rho)}(\infty)$.

(c) When $b$ is eventually nonnegative or nonpositive, the converse of (b) remains valid; that is, if $\widetilde{m} \in R_{\rho /(2-\rho)}(\infty)$ (or, equivalently, $\widetilde{M} \in R_{2 /(2-\rho)}(\infty)$ ), then $W \in R_{\rho-1}(\infty)$.

Note that $\widetilde{m} \in R_{\rho /(2-\rho)}(\infty)$ and $\widetilde{M} \in R_{2 /(2-\rho)}(\infty)$ are equivalent thanks to the monotone density theorem we mentioned above.

Proof

(a) If $W \in R_{\rho-1}(\infty)$, then $s^{\prime}=1 / W \in R_{1-\rho}(\infty)$ and hence we have $s \in R_{2-\rho}(\infty)$, which also implies that $s^{-1} \in R_{1 /(2-\rho)}(\infty)$ so that $s^{\prime} \circ s^{-1} \in R_{(1-\rho) /(2-\rho)}(\infty)$. Therefore, by (2.7) we have that $\widetilde{m}^{\prime}=2 /\left\{s^{\prime} \circ s^{-1}\right\}^{2} \in R_{-2(1-\rho) /(2-\rho)}(\infty)$.

Conversely, suppose that $\widetilde{m}^{\prime} \in R_{-(2-2 \rho) /(2-\rho)}(\infty)$. Since $\sqrt{\widetilde{m}^{\prime}} \in$ $R_{-(1-\rho) /(2-\rho)}(\infty)$, we have that $\int_{x_{0}}^{x} \sqrt{\widetilde{m}^{\prime}(u)} d u \in R_{1-(1-\rho) /(2-\rho)}(\infty)=$ $R_{1 /(2-\rho)}(\infty)$, and hence $s \in R_{2-\rho}(\infty)$ by (2.9). Combining this with the assumption that $\widetilde{m}^{\prime} \in R_{-(2-2 \rho) /(2-\rho)}(\infty)$ we obtain that $\widetilde{m}^{\prime} \circ s \in R_{-(2-2 \rho)}(\infty)$. Thus, by (2.8), we can deduce $s^{\prime} \in R_{1-\rho}(\infty)$, and therefore we conclude that $W=1 / s^{\prime} \in$ $R_{\rho-1}(\infty)$.

(b) This is an immediate consequence of (a).

(c) In view of (a), we need only to derive $\widetilde{m}^{\prime} \in R_{-(2-2 \rho) /(2-\rho)}(\infty)$ from $\widetilde{m} \in R_{\rho /(2-\rho)}(\infty)$. To this end it suffices to see that $\widetilde{m}^{\prime}$ is eventually monotone. 
Since $b$ is eventually nonnegative or nonpositive, $m^{\prime}$ is eventually monotone and, hence, so is $\widetilde{m}^{\prime}$ because $\widetilde{m}^{\prime}(x)=\frac{1}{2}\left\{m^{\prime}\left(s^{-1}(x)\right)\right\}^{2}$, which follows from (2.10) by the relation $s^{\prime}=2 / m^{\prime}$.

We have a similar result for $\rho>2$.

\section{THEOREM 3.2}

Let $\rho>2$, and let $b \in \mathbb{B}$. For simplicity we choose $c=\infty$ so that $\ell(=s(\infty))=0$.

(a) $W \in R_{\rho-1}(\infty)$ if and only if $\widetilde{m}^{\prime} \in R_{-(2-2 \rho) /(2-\rho)}(\infty)$.

(b) $W \in R_{\rho-1}(\infty)$ implies that $\widetilde{m} \in R_{\rho /(2-\rho)}(0)$ and $\widetilde{M} \in R_{2 /(2-\rho)}(0)$.

(c) The converse of (b) remains valid when $b$ is eventually nonnegative.

Theorems 3.1 and 3.2 say that if $W(x) \sim x^{\rho-1} L(x)$, then $\widetilde{m}(x) \sim x^{\rho /(2-\rho)} L_{1}(x)$ for some suitably chosen slowly varying $L_{1}(x)$. However, the relationship between $L_{1}(x)$ and $L(x)$ is rather complicated and it is not practical to try to write down explicitly even if $L(x)$ is a popular function such as $\log x$. This inconvenience may be removed if we treat $\widetilde{M}$ instead of $\widetilde{m}$ itself as follows. (Recall that (3.4) below is equivalent to Condition 3.1 (see Proposition 3.1).)

\section{THEOREM 3.3}

Let $0<\rho \neq 2$, and let $L(x)$ be slowly varying at $\infty$. Furthermore, when $\rho>2$, we normalize $s(x)$ so that $\ell(=s(\infty))=0$ (and hence $\widetilde{M}^{-1}(x)<0 \quad(x<0)$ ).

(a) If

$$
W(x) \sim W_{\rho}(x) L(x) \quad(x \rightarrow \infty)
$$

then

$$
\widetilde{M}^{-1}(x) \sim \widetilde{M}_{\rho}^{-1}(x) / L(\sqrt{x}) \quad(x \rightarrow \infty) .
$$

(b) Conversely, (3.5) implies (3.4) when b(x) is eventually nonpositive or nonnegative.

Here, when $\rho>2$, note that $\widetilde{M}^{-1}(x)$ and $\widetilde{M}_{\rho}^{-1}(x)$ are negative on $(-\infty, 0)$. But we use the notation $f(x) \sim g(x)$ when $f(x) / g(x) \rightarrow 1$ even if $f$ and $g$ are negative.

Proof

Case I: $0<\rho<2$. (a) Since $s^{\prime}(x)=1 / W(x)$ and $m^{\prime}(x)=2 W(x)$, (3.4) implies

$$
s^{\prime}(x) \sim x^{1-\rho} / L(x), \quad m^{\prime}(x) \sim 2 x^{\rho-1} L(x) \quad(x \rightarrow \infty) .
$$

Therefore (see, e.g., [1, p. 28])

$$
s(x) \sim \frac{1}{2-\rho} x^{2-\rho} / L(x), \quad m(x) \sim \frac{2}{\rho} x^{\rho} L(x) \quad(x \rightarrow \infty) .
$$


Thus, we see that $s^{-1}(x) \in R_{1 /(2-\rho)}(\infty)$ as well as that

$$
m(x) \sim \frac{2}{\rho(2-\rho)} \frac{x^{2}}{s(x)} .
$$

Therefore,

$$
\widetilde{m}(x)\left(=m\left(s^{-1}(x)\right)\right) \sim \frac{2}{\rho(2-\rho)} \frac{\left(s^{-1}(x)\right)^{2}}{x} \in R_{\{2 /(2-\rho)\}-1}(\infty) .
$$

Integrating both sides of (3.7) we see that

$$
\widetilde{M}(x) \sim \frac{1}{\rho}\left(s^{-1}(x)\right)^{2} \quad(x \rightarrow \infty) .
$$

(Recall that $\int_{1}^{x} f(u) d u \sim x f(x) / \gamma$ if $f \in R_{\gamma-1}(\infty)(\gamma>0)$.)

Considering the inverse functions of both sides of (3.8), we derive

$$
\widetilde{M}^{-1}(x) \sim s(\sqrt{\rho x}) \sim s_{\rho}(\sqrt{\rho x}) / L(\sqrt{x}),
$$

because $s(x) \sim s_{\rho}(x) / L(x)$ (see (3.6)). Note that it holds that

$$
\widetilde{M}_{\rho}^{-1}(x)=s_{\rho}(\sqrt{\rho x}),
$$

which can of course be shown directly, but it can easily be obtained as a special case with $L(x)=1$ in (3.9). Therefore, (3.9) implies (3.5).

(b) If (3.5) holds, then $\widetilde{M}^{-1} \in R_{(2-\rho) / 2}(\infty)$ so that $\widetilde{M} \in R_{2 /(2-\rho)}(\infty)$ and hence

$$
\widetilde{m} \in R_{\{(2-\rho) / 2\}-1}(\infty)=R_{\rho /(2-\rho)}(\infty) .
$$

By the assumption that $b(x)$ is eventually nonnegative or nonpositive, we can apply Theorem 3.1(c) to see that

$$
W \in R_{\rho-1}(\infty) .
$$

This means that $W(x)=x^{\rho-1} L_{0}(x)\left(=W_{\rho}(x) L_{0}(x)\right)$ for some slowly varying $L_{0}(x)$. It remains only to show that $L_{0}(x) \sim L(x)$. To this end we apply (a): $W(x)=W_{\rho}(x) L_{0}(x)$ implies that

$$
\widetilde{M}^{-1}(x) \sim \widetilde{M}_{\rho}^{-1}(x) / L_{0}(\sqrt{x}) \quad(x \rightarrow \infty) .
$$

Comparing this with the assumption (3.5) we have that $L_{0}(x) \sim L(x)(x \rightarrow \infty)$.

Case II: $\rho>2$. The proof is essentially the same as in Case I.

(a) Since $s^{\prime}(x)=1 / W(x)$ and $m^{\prime}(x)=2 W(x)$, (3.4) implies that

$$
s^{\prime}(x) \sim x^{1-\rho} / L(x), \quad m^{\prime}(x) \sim 2 x^{\rho-1} L(x) \quad(x \rightarrow \infty) .
$$

Therefore, by the well-known property of slowly varying functions,

$$
\begin{aligned}
-s(x) & =\int_{x}^{\infty} s^{\prime}(u) d u \sim \frac{1}{\rho-2} x^{2-\rho} / L(x), \\
m(x) & \sim \frac{2}{\rho} x^{\rho} L(x) \quad(x \rightarrow \infty) .
\end{aligned}
$$


Note that $s(x)<0(x>0)$ and $s(\infty)(=\ell)=0$. It also holds that $-s(x) \in$ $R_{2-\rho}(\infty)$ so that $s^{-1}(-y) \in R_{1 /(2-\rho)}(0)$. So we have that

$$
m(x) \sim \frac{2}{\rho(\rho-2)} \cdot \frac{x^{2}}{-s(x)} \quad(x \rightarrow \infty) .
$$

Now changing the variable $(y=-s(x))$, we have that

$$
\widetilde{m}(-y)=m\left(s^{-1}(-y)\right) \sim \frac{2}{\rho(\rho-2)} \frac{\left(s^{-1}(-y)\right)^{2}}{y} \quad(y \downarrow 0) .
$$

Since $-s^{-1}(-y) \in R_{1 /(2-\rho)}(0)$ as we mentioned above, (3.11) means that

$$
\widetilde{m}(-y) \in R_{\{2 /(2-\rho)\}-1}(0) .
$$

As is well known it holds in general that $\int_{x}^{1} f(u) d u \sim x f(x) / \gamma(x \downarrow 0)$ if $f \in$ $R_{\gamma-1}(0)$ and if $\gamma>0$, so we have from (3.11) and (3.12) that

$$
\widetilde{M}(-y) \sim(2-\rho) y \widetilde{m}(-y) \quad(y \downarrow 0) .
$$

Combining this with (3.11) we can deduce that

$$
\widetilde{M}(-y) \sim \frac{2}{\rho}\left(s^{-1}(-y)\right)^{2} \quad(y \downarrow 0) .
$$

Noting that $\widetilde{M}(-y) \in R_{2 /(2-\rho)}(0)$ (negative index) consider the inverse functions of both sides. Then we have that

$$
\widetilde{M}^{-1}(-x) \sim-s(\sqrt{(\rho / 2) x}) \sim-s_{\rho}(\sqrt{(\rho / 2) x}) / L(\sqrt{x}), \quad x \rightarrow \infty,
$$

because $s(x) \sim s_{\rho}(x) / L(x)$ (see (3.10)). Note that for the Bessel process it holds that

$$
\widetilde{M}_{\rho}^{-1}(x)=-s_{\rho}(\sqrt{(\rho / 2) x}),
$$

which can of course be shown directly, but it can easily be obtained as a special case with $L(x)=1$ in (3.13). Therefore, (3.13) implies (3.5).

The proof of (b) for the case $\rho>0$ is essentially the same as that for the case $0<\rho<2$.

We next study the case $\rho=2$, which we excluded in Theorem 3.3.

Let $L \in R_{0}(\infty)$. Then, $L^{*} \in R_{0}(\infty)$ is called a de Bruijin conjugate of $L$ if and only if $g(x):=x L^{*}(x)$ is an asymptotic inverse of $f(x):=x L(x)$ (i.e., $f(g(x)) \sim g(f(x)) \sim x(x \rightarrow \infty))$. A de Bruijin conjugate is uniquely determined up to asymptotic equivalence (see [1, p. 29]). Typical examples are that if $L(x) \sim$ $\log x$, then $L^{*}(x) \sim 1 / \log x$; and if $L(x)=C$, then $L^{*}(x)=1 / C$.

THEOREM $3.4(\rho=2)$

Let $L \in R_{0}(\infty)$, and let $L_{1}^{*} \in R_{0}(\infty)$ be a de Bruijin conjugate of $L_{1}(x):=L\left(x^{2}\right)$. Then, the following conditions are equivalent:

$$
W(x) \sim W_{\rho}(x) L(x)=x L(x) \quad(x \rightarrow \infty)
$$




$$
\left(\widetilde{m}^{-1}\right)^{\prime}(x) \sim \frac{L_{1}^{*}(x)}{2 x} \quad(x \rightarrow \infty)
$$

\section{REMARK 3.1}

Theorem 3.4 may look quite different from Theorem 3.3 in appearance, but in fact they are essentially the same. Indeed, we can rewrite (3.15) as follows:

$$
\frac{L(\sqrt{\lambda})}{\lambda}\left\{\widetilde{M}^{-1}(\lambda x)-\widetilde{M}^{-1}(\lambda)\right\} \rightarrow \log x, \quad x>0, \lambda \rightarrow \infty,
$$

which is similar to (3.5). Since we shall not use this fact in the rest of the article we shall not give the proof here. For the proof we need some knowledge of de Haan's class $\Gamma$ (see $[1$, Section 3.10]).

\section{Proof of Theorem 3.4}

We first remark that

$$
\left(\widetilde{m}^{-1}(x)\right)^{\prime}=\frac{s^{\prime}\left(m^{-1}(x)\right)}{m^{\prime}\left(m^{-1}(x)\right)}=\frac{2}{\left\{m^{\prime}\left(m^{-1}(x)\right)\right\}^{2}}=2\left\{\left(m^{-1}(x)\right)^{\prime}\right\}^{2} .
$$

Now let us prove first that (3.15) implies (3.14).

Let $\psi(x)=x L_{1}(x)$ so that $\psi^{-1}(x)=x L_{1}^{*}(x)$. By (3.16), we see that (3.15) implies that

$$
\left(m^{-1}(x)\right)^{\prime}=\frac{1}{\sqrt{2}} \sqrt{\widetilde{m}^{-1}(x)^{\prime}} \sim \frac{1}{2} \sqrt{\frac{L_{1}^{*}(x)}{x}} \quad(x \rightarrow \infty) .
$$

Therefore, integrating both sides we have that

$$
m^{-1}(x) \sim \sqrt{x L_{1}^{*}(x)}=\sqrt{\psi^{-1}(x)} \quad(x \rightarrow \infty) .
$$

Taking the inverse functions of both sides we obtain that

$$
m(x) \sim \psi\left(x^{2}\right) \sim x^{2} L_{1}\left(x^{2}\right)=x^{2} L(x) \quad(x \rightarrow \infty) .
$$

Now from (3.19) we derive that

$$
m^{\prime}(x) \sim 2 x L(x) \quad(x \rightarrow \infty) .
$$

Thus we have that

$$
W(x)\left(=\frac{1}{2} m^{\prime}(x)\right) \sim x L(x) .
$$

Precisely speaking, it is not clear that (3.19) implies (3.20) unless $m^{\prime}$ is monotone. However, we can easily justify it by the following argument. By (3.18) and (3.19) we have that

$$
m^{\prime}(x)=\frac{1}{\left(m^{-1}\right)^{\prime}(m(x))} \in R_{1}(\infty) .
$$

Therefore, $m^{\prime}(x)=x L_{2}(x)$ for a slowly varying $L_{2}(x)$. But this implies that $m(x) \sim(1 / 2) x^{2} L_{2}(x)$. Comparing this with (3.19) we see that in fact $L_{2}(x) \sim$ $2 L(x)$ so that $(3.20)$ holds. 
Let us prove that, conversely, (3.14) implies (3.15). Since $m^{\prime}(x)=2 W(x)$, (3.14) implies $m^{\prime} \in R_{1}(\infty)$ and hence $m \in R_{2}(\infty)$ so that $m^{-1} \in R_{1 / 2}(\infty)$. Therefore, $m^{\prime}\left(m^{-1}(x)\right) \in R_{1 / 2}(\infty)$, which implies that $\left(m^{-1}(x)\right)^{\prime} \in R_{-1 / 2}(\infty)$. Thus by (3.16)

$$
\left(\widetilde{m}^{-1}(x)\right)^{\prime}=2\left\{\left(m^{-1}(x)\right)^{\prime}\right\}^{2} \in R_{-1}(\infty) .
$$

That is, $\left(\widetilde{m}^{-1}\right)^{\prime}(x)=L_{3}(x) / x$ for some slowly varying $L_{3}$. It remains to show that $L_{3}(x) \sim(1 / 2) L_{1}^{*}(x)$. However, this is clear by the argument we used above because we already have proved that (3.15) implies (3.14).

\section{REMARK 3.2}

So far we have assumed that $V(1)=0$ (so that $W(1)=1$ ) in order to avoid the ambiguity of a multiplicative constant in the definition of $\widetilde{M}(x)$ and others. However, in fact, this does not affect at all the assertion of Theorem 3.3. Indeed, let us replace $W(x)$ by $W_{*}(x):=c W(x)$. Then $s, m, \widetilde{m}$, and $\widetilde{M}$ will be replaced by $m_{*}(x)=c m(x), s_{*}(x)=(1 / c) s(x), \widetilde{m}(x)=c \widetilde{m}(c x)$, and $\widetilde{M}_{*}(x)=\widetilde{M}(c x)$, respectively. Therefore, for instance, (3.4) and (3.5) are written as

$$
W_{*}(x) \sim W_{\rho}(x) c L(x) \quad(x \rightarrow \infty)
$$

and

$$
\widetilde{M}_{*}^{-1}(x) \sim \frac{1}{c} \widetilde{M}_{\rho}^{-1}(x) / L(\sqrt{x}) \quad(x \rightarrow \infty),
$$

respectively. Therefore, replacing $c L(x)$ by $L(x)$, we have the desired result. Thus, for example, when we apply Theorem 3.3, we may replace $V(x)$ by

$$
V_{*}(x):=\int_{0}^{x} b(u) d x, \quad x>0,
$$

if the integral makes sense.

\section{Asymptotic behavior of the transition density}

Let $p(t, x, y)$ be the transition density with respect to the speed measure $d m(x)$. It is known that $p(t, x, y)$ is symmetric in $x-y$ and has a version which is continuous in $(t, x, y)$ (see Section 5 for details). The meaning of $p(t, 0,0)$ is not clear unless the boundary 0 is regular, but we conventionally define $p(t, 0,0)=p(t,+0,+0)$.

One of the main theorems of the present article is the following. The proof will be given in Section 5 .

\section{THEOREM 4.1}

Let $\rho>0$, and suppose that Condition 3.1 is satisfied. When $\rho=2$ we assume, in addition, that 0 is of limit-circle type. Then, for all $x, y \geq 0$,

$$
p(t, x, y) \sim \frac{1}{2^{\rho / 2} \Gamma(\rho / 2)} \frac{1}{\sqrt{t}^{\rho} L(\sqrt{t})} \quad(t \rightarrow \infty),
$$


where

$$
L(t)=e^{\bar{\eta}} \exp \int_{1}^{t} \frac{\varepsilon(x)}{x} d x .
$$

Here, we say that the left endpoint $-\infty$ is of limit-circle type (in Wely's boundary classification) if

$$
\int_{-\infty}^{-A} x^{2} d \widetilde{m}(x)<\infty \quad(\exists A) .
$$

A sufficient condition for (4.3) is (2.5) with $C<3$ because (4.3) can be rewritten as

$$
\int_{-\infty}^{-A} \widetilde{M}(x) d x<\infty \quad(\exists A) .
$$

In fact, this additional assumption in the case $\rho=2$ is only a technical one and can be removed, though we shall not go into the details here.

\section{REMARK 4.1}

(a) The values of $b(x)$ on the interval $(0,1]$ do not play any role in Theorem 4.1 (as long as (2.1) is satisfied). This is simply because $p(t, x, y)$ is the density with respect to $d m(x)=2 e^{V(x)} d x$.

(b) In the definition of $V(x)$ from (2.2), the lower bound of the integral is not essential. Let us replace $V(x)$ by $V(x)+a(a \in \mathbb{R})$. Then $m(x)$ and $p(t, x, y)$ should also be replaced by $e^{a} m(x)$ and $e^{-a} p(t, x, y)$, respectively. This implies the following fact: if we denote by $p_{c}(t, x, y)$ the transition density with respect to $m_{c}(d x):=2 \exp \left(\int_{c}^{x} b(u) d u\right) d x$, then (4.6) should be replaced by

$$
p_{c}(t, x, y) \sim \frac{1}{2^{\rho / 2} \Gamma(\rho / 2)} \frac{1}{\sqrt{t}} e^{-\int_{c}^{\sqrt{t}} b(u) d u} \quad(t \rightarrow \infty) .
$$

Since $t^{\rho-1} L(t) \sim W(t)$, Theorem 4.1 may also be written as follows.

\section{THEOREM 4.2}

Suppose that $W \in R_{\rho-1}(\infty)$ ( $\left.\rho>0\right)$. When $\rho=2$ we assume, in addition, that the left boundary 0 is of limit-circle type. Then, for all $x, y \geq 0$,

$$
p(t, x, y) \sim \frac{1}{2^{\rho / 2} \Gamma(\rho / 2)} \frac{1}{\sqrt{t} W(\sqrt{t})} \quad(t \rightarrow \infty) .
$$

The converse of Theorem 4.1 holds under a mild condition as follows.

\section{THEOREM 4.3}

Let $\rho>0$, and let $L(x)$ be a slowly varying function with the canonical representation (3.2) with measurable $\varepsilon(x) \rightarrow 0$ and $c(x) \rightarrow c>0 \quad(x \rightarrow \infty)$. When $\rho=2$ we additionally assume that 0 is of limit-circle type. We further assume that $b(x)$ 
is eventually nonnegative or nonpositive. If, for some $a \geq 0$,

$$
p(t, a, a) \sim \frac{1}{2^{\rho / 2} \Gamma(\rho / 2)} \frac{1}{\sqrt{t}^{\rho} L(\sqrt{t})} \quad(t \rightarrow \infty),
$$

then Condition 3.1 is satisfied with

$$
\eta(x)=b(x)-\frac{\rho-1+\varepsilon(x)}{x}
$$

and $\bar{\eta}=\log c$.

\section{EXAMPLE 4.1}

Let $\rho>0$, and let $\beta \in \mathbb{R}$. If

$$
b(x)=\frac{\rho-1}{x}+1_{(e, \infty)}(x) \frac{\beta}{x \log x}+\eta(x), \quad x \geq 1,
$$

with $\int_{1}^{\infty-} \eta(t) d t=\bar{\eta}$, then

$$
L(x) \sim e^{\bar{\eta}} \exp \int_{e}^{x} \frac{\beta d u}{u \log u}=e^{\bar{\eta}}(\log x)^{\beta}, \quad x>e,
$$

and therefore,

$$
p(t, x, y) \sim \frac{1}{2^{\rho / 2} \Gamma(\rho / 2)} \frac{e^{-\bar{\eta}}}{\sqrt{t}^{\rho}(\log \sqrt{t})^{\beta}} \quad(t \rightarrow \infty) .
$$

The converse is also true when $b(x)$ satisfies the technical conditions in Theorem 4.3.

\section{EXAMPLE 4.2}

Let $\rho>0$, let $\beta \in \mathbb{R}$, and let $0<\gamma<1$. If

$$
b(x)=\frac{\rho-1}{x}+1_{(e, \infty)}(x) \frac{\beta \gamma}{x(\log x)^{1-\gamma}}+\eta(x), \quad x \geq 1,
$$

with $\int_{1}^{\infty-} \eta(t) d t=\bar{\eta}$, then

$$
L(x) \sim e^{\bar{\eta}-\beta} e^{\beta(\log x)^{\gamma}} \quad(x \rightarrow \infty),
$$

and therefore,

$$
p(t, x, y) \sim \frac{1}{2^{\rho / 2} \Gamma(\rho / 2)} \frac{e^{-\bar{\eta}+\beta}}{\sqrt{t}^{\rho}} e^{-\beta(\log \sqrt{t})^{\gamma}} \quad(t \rightarrow \infty) .
$$

The following corollary is only a special case of Theorems 4.1 and 4.3 but might be of interest in itself.

\section{COROLLARY 4.1}

Let $\rho>0$. If $\rho=2$, then we additionally assume that 0 is of limit-circle type.

(a) If

$$
b(x)=\frac{\rho-1}{x}+\eta(x) \quad \text { and } \quad \bar{\eta}=\lim _{A \rightarrow \infty} \int_{1}^{A} \eta(x) d x \in \mathbb{R},
$$


then, for all $x, y \geq 0$,

$$
p(t, x, y) \sim \frac{e^{-\bar{\eta}}}{2^{\rho / 2} \Gamma(\rho / 2)} \frac{1}{\sqrt{t}^{\rho}} \quad(t \rightarrow \infty) .
$$

(b) Conversely, if, for some $a>0$,

$$
p(t, a, a) \sim \frac{e^{-\bar{\eta}}}{2^{\rho / 2} \Gamma(\rho / 2)} \frac{1}{\sqrt{t}^{\rho}} \quad(t \rightarrow \infty)
$$

and if $b(x)$ is eventually nonnegative or nonpositive, then (4.9) holds.

\section{REMARK 4.2}

In Theorem 4.3 the assumption that " $b(x)$ is eventually nonnegative or nonpositive" is crucial. Without this restriction, the assertion does not hold in general. Here is a counterexample. Let

$$
b(x)=\sin 2 \pi x \quad(x>0)
$$

so that $\rho=1$ and $\eta=\sin 2 \pi x$. Or more generally, let $b(x)$ be a periodic function with period 1 such that

$$
\int_{0}^{1} b(x) d x=0 .
$$

Then $W(x)=\exp \int_{1}^{x} b(u) d u$ has period 1 . Therefore,

$$
s(x)=\int_{c}^{x} \frac{d u}{W(u)} \sim A x \quad(x \rightarrow \infty), A=\int_{0}^{1} e^{-b(u)} d u,
$$

and

$$
m(x)=2 \int_{0}^{x} W(u) d u \sim 2 B x \quad(x \rightarrow \infty), B=\int_{0}^{1} e^{b(u)} d u
$$

so that

$$
\widetilde{m}(x)=m\left(s^{-1}(x)\right) \sim \frac{2 B}{A} x \quad(x \rightarrow \infty) .
$$

Therefore, we see from Theorem A that $p(t, x, y) \sim$ const $\cdot t^{-1 / 2}$, but (4.9) fails because the left-hand side oscillates. This phenomenon might be related to Remark 2.1.

\section{Proofs of Theorems 4.1 and 4.3}

The proofs of Theorems 4.1 and 4.3 are based on the following result of Kotani from [11] and [10] (see also [7], [9]) where the relationship between $\widetilde{m}(x)$ and the spectral function $\sigma(\xi)$ is discussed as an application of Krein-type spectral theory. We shall not go into the details of the definition of the spectral function since the only thing we need here is the following fact.

To begin, we stress that we assumed the condition (2.1) so that

$$
\int_{-\infty}^{-A} \widetilde{M}^{k}(x) d x<\infty, \quad \exists A>0,
$$


for sufficiently large $k \geq 1$ (see Lemma 2.1), so that $\widetilde{m}$ is within the framework of Kotani (see [11]). Let $\varphi_{\lambda}(x)(x<\ell)$ be the unique solution of

$$
\mathcal{L} u=-\lambda u, \quad u(-\infty)=1,
$$

or, precisely, of the following integral equation:

$$
\varphi_{\lambda}(x)=1-\lambda \int_{-\infty}^{x}(x-y) \varphi_{\lambda}(y) d \widetilde{m}(y) \quad(x<\ell) .
$$

Then the transition density of the diffusion with respect to the speed measure $d m(x)$ is given by

$$
p(t, x, y)=\int_{-0}^{\infty} e^{-t \lambda} \varphi_{\lambda}\left(s^{-1}(x)\right) \varphi_{\lambda}\left(s^{-1}(y)\right) d \sigma(\lambda), \quad t>0 .
$$

Since $p(t, x, y)$ is continuous in $(t, x, y)$, letting $x, y \rightarrow+0$ we see that

$$
p(t, 0,0)=\int_{-0}^{\infty} e^{-t \lambda} d \sigma(\lambda), \quad t>0 .
$$

\section{THEOREM A ([11]; SEE ALSO [9, THEOREM 2.4])}

Let $0<\alpha<1$, and let $\varphi \in R_{\alpha-1}(0)$ so that $\varphi^{-1} \in R_{1 /(\alpha-1)}(\infty)$. Then, the following are equivalent:

$$
\begin{aligned}
& \widetilde{m}(x) \sim \frac{C_{\alpha}}{x \varphi^{-1}(x)} \quad(x \rightarrow \infty), \quad C_{\alpha}=\left(\frac{1-\alpha}{\alpha}\right)^{\alpha /(1-\alpha)} \\
& \sigma(\xi) \sim \frac{\alpha^{2 \alpha}}{\Gamma(1+\alpha)^{2}} \xi \varphi(\xi) \quad(\xi \rightarrow+0) .
\end{aligned}
$$

\section{COROLLARY 5.1}

Let $0<\alpha<1$. Then $\sigma \in R_{\alpha}(0)$ if and only if $\tilde{m} \in R_{\alpha /(1-\alpha)}(\infty)$.

The following theorem was proved in [9] for the case $1<\alpha<2$, and the general case is due to [11] and [10].

\section{THEOREM B}

Let $\alpha>1$, and let $\varphi \in R_{\alpha-1}(0)$ so that $\varphi^{-1} \in R_{1 /(\alpha-1)}(0)$. We translate $\widetilde{m}$ so that $\ell=0$; that is, $\widetilde{m}(x)<\infty(x<0)$ and $\widetilde{m}(x)=\infty(x \geq 0)$.

(a) If

$$
\widetilde{m}(-x) \sim \frac{C_{\alpha}}{x \varphi^{-1}(x)} \quad(x \rightarrow+0), \quad C_{\alpha}=\left(\frac{\alpha-1}{\alpha}\right)^{\alpha /(1-\alpha)},
$$

then

$$
\sigma(\xi) \sim \frac{\alpha^{2 \alpha}}{\Gamma(1+\alpha)^{2}} \xi \varphi(\xi) \quad(\xi \rightarrow+0) .
$$

(b) Conversely, (5.6) implies (5.5) provided that (2.6) is satisfied for some $k>\alpha-1$. 


\section{COROLLARY 5.2}

Let $\alpha>1$. Then, $\sigma \in R_{\alpha}(0)$ if and only if $\widetilde{m}(-x) \in R_{\alpha /(1-\alpha)}(0)$.

For the case $\alpha=1$ we have the following, which is a special case of [8, Theorem 2].

\section{THEOREM C}

We assume that the left endpoint $-\infty$ is of limit-circle type and confine ourselves to the case where $\widetilde{m}$ has continuous derivative $\widetilde{m}^{\prime}$.

Let $L_{1} \in R_{0}(\infty)$, and let $L_{1}^{*}$ be its de Bruijin conjugate.

(a) If

$$
\left(\widetilde{m}^{-1}(x)\right)^{\prime} \sim \frac{L_{1}^{*}(x)}{x} \quad(x \rightarrow \infty)
$$

then

$$
\sigma(\xi) \sim \frac{\xi}{L_{1}(1 / \xi)} \quad(\xi \rightarrow+0)
$$

(b) Conversely, (5.8) implies (5.7) when $\widetilde{m}^{\prime}$ is eventually monotone.

For our present use, let us rewrite Theorems A and B as follows.

\section{PROPOSITION 5.1}

(a) Let $0<\alpha<1$. Then $\widetilde{M}^{-1} \in R_{1-\alpha}(\infty)$ if and only if $\sigma \in R_{\alpha}(0)$. When one of them (hence both) holds, we have that

$$
\widetilde{M}^{-1}(x) \sim \frac{1}{\alpha^{\alpha}(1-\alpha) \Gamma(1+\alpha)^{2}} x \sigma(1 / x) \quad(x \rightarrow \infty) .
$$

(b) Let $\alpha>1$, and let $\ell=0$. Then $-\widetilde{M}^{-1} \in R_{1-\alpha}(\infty)$ if and only if $\sigma \in$ $R_{\alpha}(0)$. Furthermore, when one of them (hence both) holds, we have that

$$
-\widetilde{M}^{-1}(x) \sim \frac{1}{\alpha^{\alpha}(\alpha-1) \Gamma(1+\alpha)^{2}} x \sigma(1 / x) \quad(x \rightarrow \infty) .
$$

Here, note that $\widetilde{M}^{-1}(x)$ is positive or negative according to whether $\alpha<1$ or $\alpha>1$.

\section{Proof}

Since the proofs (a) and (b) are essentially the same we shall prove the case $0<\alpha<1$ only. By the usual properties of regularly varying functions $\widetilde{M}^{-1} \in$ $R_{1-\alpha}(\infty)$ if and only if $\widetilde{M} \in R_{1 /(1-\alpha)}(\infty)$, which is equivalent to $\widetilde{m} \in$ $R_{\alpha /(1-\alpha)}(\infty)$. But this is also equivalent to $\sigma(\xi) \in R_{\alpha}(0)$ by Corollary 5.1, which proves the first half of the assertion. To see the latter half suppose that $\sigma(\cdot) \in$ $R_{\alpha}(0)$, and choose $\varphi(\cdot) \in R_{\alpha-1}(0)$ so that (5.4) (and hence (5.3)) holds. Then

$$
\sigma(1 / x) \sim \frac{\alpha^{2 \alpha}}{\Gamma(1+\alpha)^{2}} \frac{\varphi(1 / x)}{x} \quad(x \rightarrow \infty)
$$


and

$$
\widetilde{M}(x)=\int_{-\infty}^{x} u d \widetilde{m}(u) \sim(1-\alpha) x \widetilde{m}(x) \sim(1-\alpha) C_{\alpha} \frac{1}{\varphi^{-1}(x)} \quad(x \rightarrow \infty),
$$

which is equivalent to

$$
\begin{aligned}
\widetilde{M}^{-1}(x) & \sim \varphi\left(\frac{1}{(1-\alpha) C_{\alpha} x}\right) \\
& \sim\left((1-\alpha) C_{\alpha}\right)^{1-\alpha} \varphi\left(\frac{1}{x}\right) \quad(x \rightarrow \infty)
\end{aligned}
$$

by Theorem A (Theorem B for the case $\alpha>1$ ). Now compare (5.12) with (5.11).

\section{COROLLARY 5.3}

Let $\rho>0$, and let $L_{1} \in R_{0}(\infty)$.

(a) When $\rho \neq 2$, the following conditions are equivalent:

$$
\begin{aligned}
\widetilde{M}^{-1}(x) & \sim \widetilde{M}_{\rho}^{-1}(x) / L_{1}(x) \quad(x \rightarrow \infty), \\
p(t, x, y) & \sim p_{\rho}(t, 0,0) / L_{1}(t) \quad(t \rightarrow \infty), \forall x, y \geq 0, \\
p(t, 0,0) & \sim p_{\rho}(t, 0,0) / L_{1}(t) \quad(t \rightarrow \infty), \\
\sigma(\xi) & \sim \sigma_{\rho}(\xi) / L_{1}(1 / \xi) \quad(\xi \rightarrow+0),
\end{aligned}
$$

where $\sigma_{\rho}$ corresponds to $m_{\rho}$.

(b) When $\rho=2$, with the additional assumption on limit-circle type, the assertion of (a) remains valid if we replace (5.13) by

$$
\left(\widetilde{m}^{-1}(x)\right)^{\prime} \sim \frac{L_{1}^{*}(x)}{x} \quad(x \rightarrow \infty) .
$$

As we mentioned in Remark 3.1, (5.13) and (5.17) are, in fact, essentially the same.

Proof

Let $\alpha=\rho / 2$ so that $0<\alpha<1$. As a special case of (5.9), we have that

$$
\widetilde{M}_{\rho}^{-1}(x) \sim D_{\alpha} x \sigma_{\rho}(1 / x) \quad(x \rightarrow \infty) .
$$

Taking the ratio of (5.9) and (5.18), we see that

$$
\frac{\widetilde{M}^{-1}(x)}{\widetilde{M}_{\rho}^{-1}(x)} \sim \frac{\sigma(1 / x)}{\sigma_{\rho}(1 / x)} \quad(x \rightarrow \infty),
$$

which proves the equivalence of (5.13) and (5.16).

Since $p(t, 0,0)$ is the Laplace transform of $\sigma$ (see $(5.1)$ ), we have the equivalence of (5.15) and (5.16) by the well-known Karamata-Tauberian theorem. It remains to show that (5.16) implies (5.14). To this end, we go back to (5.1). 
When $\sigma(\lambda)$ varies regularly as $\lambda \rightarrow+0$, then

$$
\begin{aligned}
p(t, x, y) & =\int_{-0}^{1} e^{-t \lambda} \varphi_{\lambda}\left(s^{-1}(x)\right) \varphi_{\lambda}\left(s^{-1}(y)\right) d \sigma(\lambda)+O\left(e^{-t / 2}\right) \\
& \sim \int_{-0}^{1} e^{-t \lambda} d \sigma(\lambda), \quad t \rightarrow \infty
\end{aligned}
$$

because $\varphi_{\lambda}\left(s^{-1}(x)\right) \varphi_{\lambda}\left(s^{-1}(y)\right) \rightarrow 1$ as $\lambda \rightarrow 0$. We used here the fact that

$$
\int_{1}^{\infty} e^{-t \lambda} \varphi_{\lambda}\left(s^{-1}(x)\right) \varphi_{\lambda}\left(s^{-1}(y)\right) d \sigma(\lambda)=O\left(e^{-t / 2}\right),
$$

which follows from

$$
\left(\int_{1}^{\infty} e^{-t \lambda} \varphi_{\lambda}(u) \varphi_{\lambda}(v) d \sigma(\lambda)\right)^{2} \leq \int_{1}^{\infty} e^{-t \lambda} \varphi_{\lambda}(u)^{2} d \sigma(\lambda) \int_{1}^{\infty} e^{-t \lambda} \varphi_{\lambda}(v)^{2} d \sigma(\lambda)
$$

and

$$
\int_{1}^{\infty} e^{-t \lambda} \varphi_{\lambda}(u)^{2} d \sigma(\lambda) \leq e^{-t / 2} \int_{1}^{\infty} e^{-t \lambda / 2} \varphi_{\lambda}(u)^{2} d \sigma(\lambda) \leq e^{-t / 2} p(t / 2, u, u) .
$$

We are now ready to prove Theorems 4.1 and 4.3. In Corollary 5.3 we gave a necessary and sufficient condition for the asymptotic behavior of $p(t, x, y)$ in terms of $\widetilde{M}(x)$. On the other hand in Section 3 we studied the relationship between the asymptotic behavior of $\widetilde{M}(x)$ and that of $b(x)$. Therefore, the relationship between $p(t, x, y)$ and $b(x)$ is now clear.

\section{Proof of Theorem 4.1}

First recall that $W(x)=e^{V(x)}=x^{\rho-1} L(x)$. When $\rho \neq 2$, by Theorem 3.3 we have that

$$
\widetilde{M}^{-1}(x) \sim \widetilde{M}_{\rho}^{-1}(x) / L(\sqrt{x}) \quad(x \rightarrow \infty) .
$$

Note that if $L(x)$ varies slowly then so does $L(\sqrt{x})$. Now applying Corollary 5.3 with $L_{1}(x)=L(\sqrt{x})$, we can deduce the assertion of Theorem 4.1. Similarly we can treat the case where $\rho=2$.

Proof of Theorem 4.3

We shall see that

$$
\eta(x):=b(x)-\left(\frac{\rho-1}{x}+\frac{\varepsilon(x)}{x}\right)
$$

satisfies the desired property. Suppose that (4.7) holds. Then by Corollary 5.3 we have (5.13) with $L_{*}(x)=1 / L(\sqrt{x})$. Therefore, by Theorem 3.3(b), we have that

$$
W(x)=e^{V(x)} \sim x^{\rho-1} L(x) \quad(x \rightarrow \infty)
$$

that is,

$$
V(x)-(\rho-1) \log x-\log L(x) \rightarrow 0 \quad(x \rightarrow \infty) .
$$


Recalling the form of $V(x)$ and $L(x)$, we can rewrite (5.22) as

$$
\int_{1}^{x} b(u) d u-\left\{\int_{1}^{x} \frac{\rho-1}{t} d t+\int_{1}^{x} \frac{\varepsilon(t)}{t} d t+\log c(x)\right\} \rightarrow 0 .
$$

By the definition of $\eta(x)$ (see(5.20)) this can also be written as

$$
\int_{1}^{x} \eta(u) d u-\log c(x) \rightarrow 0 \quad(x \rightarrow \infty) .
$$

Since $c(x) \rightarrow c$ by assumption, we have that $\bar{\eta}=\log c$.

\section{REMARK 5.1}

The following form of Theorem 4.1 might be useful in some other problems. Let $\mathcal{L}=\mathcal{L}_{\rho}+q(x) \frac{d}{d x}$ with $q(x)=o(1 / x)$. Then

$$
\sigma(\xi) \sim \sigma_{\rho}(\xi) e^{-\int_{1}^{1 / \sqrt{\xi}} q(u) d u} \quad(\xi \rightarrow+0) .
$$

\section{Applications to skew Bessel processes}

Let $X=\left(X_{t}\right)_{t \geq 0}$ be a diffusion process on $\mathbb{R}$ whose canonical form of the generator is

$$
\mathcal{L}=\frac{d}{d \widetilde{m}(x)} \frac{d}{d x}, \quad-\infty<x<\infty .
$$

Let $\widetilde{m}_{+}(x)=\widetilde{m}[0, x]$, and let $\widetilde{m}_{-}(x)=\widetilde{m}[-x, 0)$ for $x>0$. The diffusion $X$ is called a skew Bessel process of dimension $0<\rho<2$ and skewness $0<p<1$ if, for some $c>0$,

$$
\begin{cases}\widetilde{m}_{+}(x)=c p^{2 /(2-\rho)} x^{\rho /(2-\rho)}, & x>0 \\ \widetilde{m}_{-}(x)=c(1-p)^{2 /(2-\rho)} x^{\rho /(2-\rho)}, & x>0 .\end{cases}
$$

This process is a generalization of the skew Brownian motion introduced in [5, p. 115] (see also [3]). It is a self-similar diffusion and appears in some limit theorems.

We may say that the diffusion $X$ (or $\widetilde{m}$ ) belongs to the domain of attraction of (6.1) if, as $x \rightarrow \infty$,

$$
\left\{\begin{array}{l}
\widetilde{m}_{+}(x) \sim p^{2 /(2-\rho)} x^{\rho /(2-\rho)} L(x), \\
\widetilde{m}_{-}(x) \sim(1-p)^{2 /(2-\rho)} x^{\rho /(2-\rho)} L(x),
\end{array}\right.
$$

for some slowly varying $L(x)$. This condition appears in some limit theorems. For example, Watanabe [13] proved that (6.2) is the necessary and sufficient condition for a generalized arcsine law to hold.

Note that (6.1) is equivalent to

$$
\left(\widetilde{M}_{+}\right)^{-1}(x)=\frac{c^{\prime}}{p} x^{(2-\rho) / 2}, \quad\left(\widetilde{M}_{-}\right)^{-1}(x)=\frac{c^{\prime}}{1-p} x^{(2-\rho) / 2}, \quad x>0,
$$

for some $c^{\prime}>0$. Therefore, it is easy to see that (6.2) is equivalent to

$$
\left(\widetilde{M}_{+}\right)^{-1}(x) \in R_{(2-\rho) / 2}(\infty)
$$


and

$$
\lim _{x \rightarrow \infty} \frac{\left(\widetilde{M}_{-}\right)^{-1}(x)}{\left(\widetilde{M}_{+}\right)^{-1}(x)}=\frac{p}{1-p} .
$$

Now let us interpret the above two conditions for the process associated with

$$
\mathcal{L}=\frac{1}{2}\left(\frac{d^{2}}{d x^{2}}+b(x) \frac{d}{d x}\right), \quad x \in \mathbb{R} .
$$

In what follows we assume that $b \in L_{\mathrm{loc}}(\mathbb{R}, d x)$. Feller's canonical form of (6.5) is obtained as follows. Define

$$
W(x)=\exp \int_{0}^{x} b(u) d u, \quad-\infty<x<\infty .
$$

Then, the scale function and the speed measure are

$$
s(x)=\int_{0}^{x} \frac{d u}{W(u)}, \quad m(x)=2 \int_{0}^{x} W(u) d u, \quad-\infty<x<\infty,
$$

and therefore $\widetilde{m}(x)=m\left(s^{-1}(x)\right)$. So according to the definition of $\widetilde{m}_{ \pm}(x)$ it holds that $\widetilde{m}_{+}(x)=\widetilde{m}(x)$ and $\widetilde{m}_{-}(x)=-\widetilde{m}(-x)$. Notice that the latter corresponds to

$$
W_{-}(x):=\exp \left(-\int_{0}^{x} b(-u) d u\right), \quad x \geq 0
$$

in place of (6.6). This can easily be understood if we recall that $\widetilde{m}_{-}$corresponds to

$$
\frac{1}{2}\left(\frac{d^{2}}{d x^{2}}-b(-x) \frac{d}{d x}\right), \quad x \geq 0
$$

THEOREM 6.1

(a) If Condition 3.1 is satisfied $($ on $[1, \infty)$ ) and if

$$
\lim _{A \rightarrow \infty} \int_{-A}^{A} b(x) d x=\log \frac{p}{1-p},
$$

then the process belongs to the domain of attraction of (6.1).

(b) The converse of (a) holds if each of $b(x)$ and $b(-x)$ is eventually nonnegative or nonpositive as $x \rightarrow \infty$.

\section{Proof}

(a) As we have seen above, it is sufficient to see that (6.3) and (6.4) are satisfied. Condition 3.1 implies that $W \in R_{\rho-1}(\infty)$. So by Theorem 3.3(a) we have (6.3). To see (6.4) first note that, by (6.9),

$$
\frac{W_{-}(x)}{W(x)}=\exp \left(-\int_{-x}^{x} b(u) d u\right) \rightarrow \frac{1-p}{p} .
$$

This implies that $W_{-}$(as well as $W$ ) is in $R_{\rho-1}(\infty)$. Then using Theorem 3.3(a) again we can deduce (6.4) (see Remark 3.2). 
(b) By Theorem 3.3(b) and Proposition 3.1 we see that Condition 3.1 is satisfied. It remains to show (6.9) or equivalently (6.10), but the latter is clear from Theorem 3.3(b).

We next study the asymptotic behavior of the transition density $p(t, x, y)$ with respect to $d m(x)=2 W(x) d x$.

\section{THEOREM 6.2}

Suppose that (6.9) is satisfied, and suppose that $W \in R_{\rho-1}(\infty)(0<\rho<2)$. Then,

$$
p(t, 0,0) \sim \frac{p}{2^{\rho / 2} \Gamma(\rho / 2)} \frac{1}{\sqrt{t} W(\sqrt{t})} \quad(t \rightarrow \infty) .
$$

Proof

Let $p_{+}(t, x, y)$ and $p_{-}(t, x, y)$ be the transition densities of the diffusions on $[0, \infty)$ with generators of

$$
\frac{d}{d \widetilde{m}_{+}(x)} \frac{d}{d x} \quad \text { and } \quad \frac{d}{d \widetilde{m}_{-}(x)} \frac{d}{d x},
$$

respectively. By Theorem 4.1 we have, as $t \rightarrow \infty$, that

$$
\begin{aligned}
& p_{+}(t, 0,0) \sim \frac{1}{2^{\rho / 2} \Gamma(\rho / 2)} \frac{1}{\sqrt{t} W(\sqrt{t})}, \\
& p_{-}(t, 0,0) \sim \frac{1}{2^{\rho / 2} \Gamma(\rho / 2)} \frac{1}{\sqrt{t} W_{-}(\sqrt{t})} .
\end{aligned}
$$

These two combined with (6.10) imply that

$$
p_{-}(t, 0,0) \sim \frac{p}{1-p} p_{+}(t, 0,0) \quad(t \rightarrow \infty) .
$$

Now let

$$
h(s)=\int_{0}^{\infty} e^{-s t} p(t, 0,0) d t, \quad s>0,
$$

and similarly let $h_{+}(s)$ and $h_{-}(s)$ correspond to $p_{+}(t, 0,0)$ and $p_{-}(t, 0,0)$, respectively. Then, as is well known, it holds that

$$
\frac{1}{h(s)}=\frac{1}{h_{+}(s)}+\frac{1}{h_{-}(s)} \text {. }
$$

Since (6.12) implies that $h_{-}(s) \sim(p /(1-p)) h_{+}(s)(s \rightarrow+0)$, we have from (6.13) that

$$
h(s) \sim p h_{+}(s) \quad(s \rightarrow+0),
$$

which proves that $p(t, 0,0) \sim p p_{+}(t, 0,0)(t \rightarrow \infty)$ thanks to the KaramataTauberian theorem. 


\section{References}

[1] N. H. Bingham, C. M. Goldie, and J. L. Teugels, Regular Variation, Encyclopedia Math. Appl. 27, Cambridge Univ. Press, Cambridge, 1987. MR 0898871. DOI 10.1017/CBO9780511721434.

[2] A. N. Borodin and P. Salminen, Handbook of Brownian Motion-Facts and Formulae, 2nd ed., Birkhäuser, Basel, 2002. MR 1912205.

DOI 10.1007/978-3-0348-8163-0.

[3] J. M. Harrison and L. A. Shepp, On skew Brownian motion, Ann. Probab. 9 (1981), 309-313. MR 0606993.

[4] K. Itô, Essentials of Stochastic Processes, Transl. Math. Monogr. 231, Amer. Math. Soc., Providence, 2006, MR 2239081.

[5] K. Itô and H. P. McKean, Jr., Diffusion Processes and Their Sample Paths, Grundlehren Math. Wiss. 125, Springer, New York, 1974. MR 0345224.

[6] J. Karamata, Sur un mode de croissance régulière. Théorèmes fondamentaux, Bull. Soc. Math. France 61 (1953), 55-62. MR 1504998.

[7] Y. Kasahara, Spectral theory of generalized second order differential operators and its applications to Markov processes, Japan. J. Math. (N.S.) 1 (1975/76), 67-84. MR 0405615.

[8] Spectral function of Krein's and Kotani's string in the class $\Gamma$, Proc. Japan Acad. Ser. A Math. Sci. 88 (2012), 173-177. MR 3004234. DOI 10.3792/pjaa.88.173.

[9] Y. Kasahara and S. Watanabe, Asymptotic behavior of spectral measures of Krein's and Kotani's strings, Kyoto J. Math. 50 (2010), 623-644. MR 2723865. DOI 10.1215/0023608X-2010-007.

[10] S. Kotani, Krein's strings with singular left boundary, Rep. Math. Phys. 59 (2007), 305-316. MR 2347790. DOI 10.1016/S0034-4877(07)80067-X.

[11] Krein's strings whose spectral functions are of polynomial growth, Kyoto J. Math. 53 (2013), 787-814. MR 3160601.

DOI 10.1215/21562261-2366094.

[12] M. Tomisaki and M. Yamazato, Limit theorems for hitting times of 1-dimensional generalized diffusions, Nagoya Math. J. 152 (1998), 1-37. MR 1659393.

[13] S. Watanabe, "Generalized arc-sine laws for one-dimensional diffusion processes and random walks" in Stochastic Analysis (Ithaca, NY, 1993), Proc. Sympos. Pure Math. 57, Amer. Math. Soc., Providence, 1995, 157-172. MR 1335470. DOI 10.1090/pspum/057/1335470.

[14] Y. Yano, On the occupation time on the half line of pinned diffusion processes, Publ. Res. Inst. Math. Sci. 42 (2006), 787-802. MR 2266997.

Kasahara: Institute of Mathematics, University of Tsukuba, Tsukuba, Ibaraki 305-8751, Japan; kasahara@math.tsukuba.ac.jp 
Kotani: Department of Mathematical Sciences, Kwansei Gakuin University, Sanda, Hyogo 669-1337, Japan; kotani@kwansei.ac.jp 